\title{
Master Manipulator Designed for Highly Articulated Robotic Instruments in Single Access Surgery
}

\author{
Piyamate Wisanuvej ${ }^{1,2}$, Gauthier Gras ${ }^{1}$, Konrad Leibrandt ${ }^{1}$, Petros Giataganas ${ }^{1}$, \\ Carlo A. Seneci ${ }^{1}$, Jindong $\mathrm{Liu}^{1}$, and Guang-Zhong Yang ${ }^{1}$, Fellow, IEEE
}

\begin{abstract}
The performance of a master-slave robotic system depends significantly on the ergonomics and the capability of its master device to correctly interface the user with the slave robot. Master manipulators generating commands in task space represent a commonly adopted solution for controlling a range of slave robots while retaining an ergonomic design. However, these devices present several drawbacks, such as requiring the use of clutching mechanics to compensate for the mismatch between slave and master workspaces, and the lack of capability to intuitively transmit important information such as specific joint limits to the user. In this paper, a novel joint-space master manipulator is presented. This manipulator emulates the kinematic structure of highly flexible surgical instruments which it is designed to control. This system uses 6 active degrees of freedom to compensate for its own weight, as well as to provide force feedback corresponding to the slave robot's joint limits. A force/torque sensor integrated at the end effector is used to relay user-generated forces and torques directly to specific joints. This is performed to counteract the friction stemming from structural constraints imposed by the kinematic design of the instruments. Finally, a usability study is carried out to test the validity of the system, proving that the instruments can be intuitively controlled even at the extremities of the workspace.
\end{abstract}

\section{INTRODUCTION}

With the technological advances of the last decades, robotic systems have become an integral part of the operating theatre in multiple hospitals around the globe. A widespread solution for the control of these systems is to use a masterslave paradigm, under which the operator's motions are relayed through a master device to the slave robot. This approach is particularly appealing in surgery, as it allows the user to benefit from robotic advantages such as motion scaling and tremor reduction, while retaining direct control over the robot motions.

A consequence of this paradigm is that the quality of a robotic system depends in large part on the quality of its master manipulator. An advanced slave system composed of highly dexterous instruments will ultimately not perform to its full potential if paired with an inadequate master system. To overcome this issue, a large number of systems opt to use master devices generating commands in task space. This allows such devices to be suitable for a large range of systems, while retaining an intuitive behaviour. Examples of master

This research is conducted with support from the Department of Health, United Kingdom and the Wellcome Trust through the Health Innovation Challenge Fund (HICF-T4-299).

${ }^{1}$ All authors are with the Hamlyn Centre for Robotic Surgery, Imperial College London, SW7 2AZ, UK. ${ }^{2}$ Piyamate Wisanuvej is also with Kasetsart University, Bangkok, Thailand.

Corresponding author: piyamate.w12@imperial.ac.uk

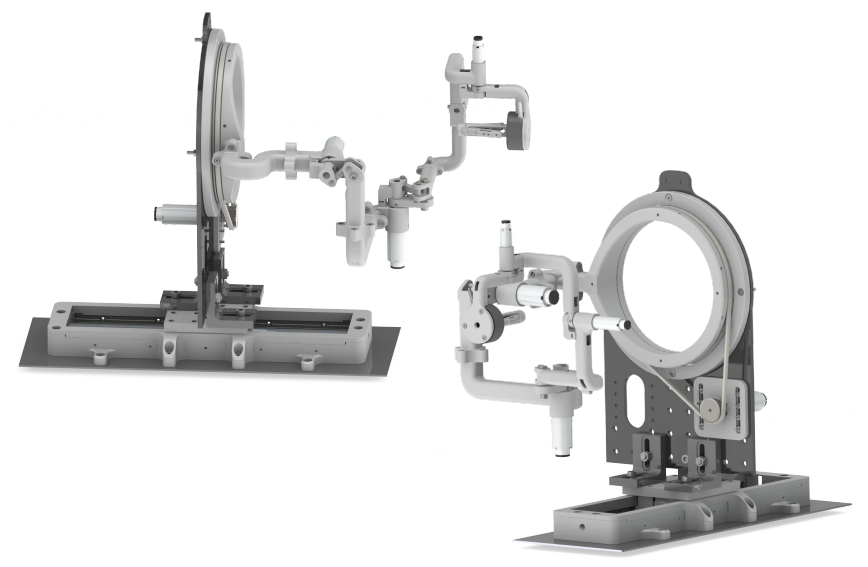

Fig. 1. Different viewpoints of the proposed master manipulator.

devices operating in task space include delta platforms such as the omega.7 and sigma.7 haptic devices (Force Dimension, Switzerland), or the master manipulator of the FLEXMIN system [1]. Other task space master manipulators include serial-link devices such as the Geomagic Touch (Geomagic, USA), and the da Vinci surgical system master manipulator (Intuitive Surgical, USA).

While master manipulators operating in task space offer advantages in terms of generality and ease of use, their lack of specificity also present some disadvantages. In previous work, the authors designed highly articulated surgical instruments with 7 degrees of freedom (DoFs) for single access surgery [2]. An inverse kinematic control scheme [3] was developed to take advantage of the flexibility of the instruments, using two omega.7 devices as master manipulators. However, due to the mismatch between the master and slave workspaces, clutching mechanics had to be implemented, slowing down the user. Furthermore, the delta platform design of the master manipulator did not easily allow the user to perceive the joint motions and limits of the instruments. As the final joint positions are the result of the optimisation process of the inverse kinematic solver, this can lead to situations where the user is unaware of which joints have reached their limits and how to work around them to reach a specific goal.

As a result, the use of a device-specific master manipulator commanding the slave robot in joint space can be an attractive alternative. In [4], a master manipulator is presented for the control of a slave robotic device in joint space. 


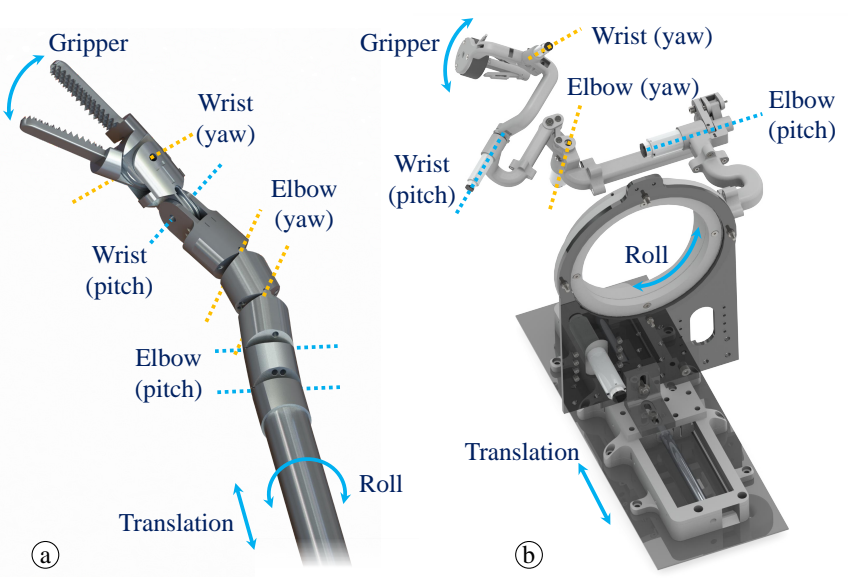

Fig. 2. Joint control mapping of (a) the highly articulated robotic instrument to (b) the master manipulator.

However, the low number of DoFs used does not make it suitably scalable for the control of highly articulated surgical instruments. A number of joint space master manipulators with a larger number of DoFs have been proposed, such as presented in [5], but do not possess active joints. This makes them unable to compensate for their own weight, or provide other forms of motion assistance. A master device with a high number of DoFs and active joints is presented in [6], but its delta structure prevents its use for the joint control of the instruments discussed. In [7] an exoskeleton-like robotic manipulator is placed on the human arm to control a surgical robot for gastrointestinal (GI) tract surgery. This passive system senses the joint-angles of the exoskeleton with rotary encoders, and hence cannot be gravity compensated or be used to give haptic feedback to the operator.

In this paper, a novel master manipulator for joint space control is presented. This device is designed for use with highly articulated instruments in Transanal Endoscopic Micro-Surgery (TEMS). The device possesses 6 active DoFs and a gripper, for a total of $7 \mathrm{DoFs}$. The design of the master manipulator allows the user to cover the entire workspace of the instruments without clutching. Furthermore, the active DoFs are used to render joint limits on the master device, granting the user an intuitive understanding of the state of the instruments. Standard master devices employ mechanical structures designed to limit the friction and resistance felt by the user. This approach is not possible here as the structure of the master device is dictated by the kinematics of the robot. To overcome this issue, a force/torque sensor is used to compensate for the excessive friction and weight presented in some joints. Lastly, a usability study is carried out to characterise the performance of the system.

\section{Manipulator Design}

The slave surgical instrument used in conjunction with the master manipulator is part of a robotic surgical system targeted for use in a TEMS operation. Full details of the overall surgical system are described in [2], [3].

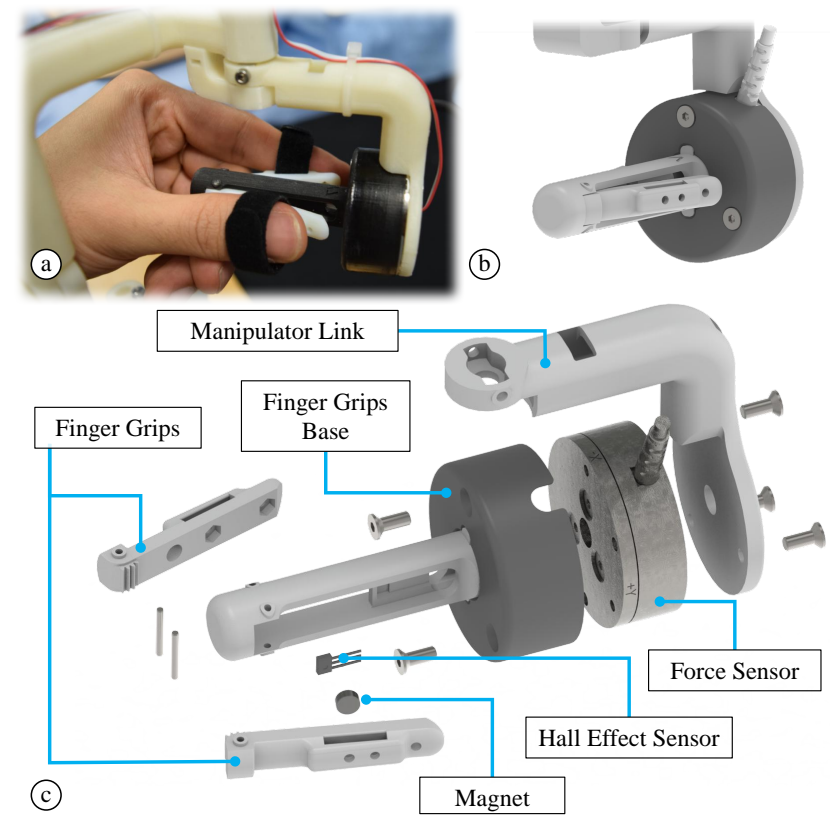

Fig. 3. Master manipulator's finger grips design; (a) operator while manipulating the finger grips, (b) CAD rendering of the finger grips, and (c) exploded view presenting the magnet and the Hall effect sensor for position sensing and the integrated force sensor to facilitate the manipulator's motion.

\section{A. Surgical Instrument}

The instrument shown in Fig. 2 (a) consists of a cylindrical shaft, articulated elbow segments, a wrist, and a gripper. This encompasses 7 active DoFs plus 2 passive DoFs which are 1:1 coupled with the active ones within the same pair (dependent joints). The dimensions and manufacturing process of the tool is iteratively optimised to accommodate the workspace and forces required by the surgical procedure [8].

\section{B. Hardware Design}

As the master manipulator is designed to work in joint space control, the kinematic structure of the device is dictated by the surgical instrument. Fig. 2 shows the correspondence between the master and slave devices. Since the manipulation of both devices is mapped directly in the joint space, no clutching is required during operation. This imposes a fixed motion scaling for the telemanipulation. The scaling factor is selected so that the overall length of the device is within the motion range of an average human forearm. This results in the scaling factor of 5 with overall length of $300 \mathrm{~mm}$.

The design of the master manipulator is presented in Fig. 1. Each active rotational joint is actuated by a DC motor with an integrated gearhead and an incremental encoder (Maxon motor, Switzerland). The reduction ratios are chosen high enough so that the motor can produce sufficient torque to counter the gravity load and render the forces to the user, but also not too high so that they can be backdriven. The translation stage is powered by a linear DC-Servomotor (Faulhaber, Germany), which can produce enough force required for friction compensation while holding the load from all the rotational joints above it. Finger grips are fitted 


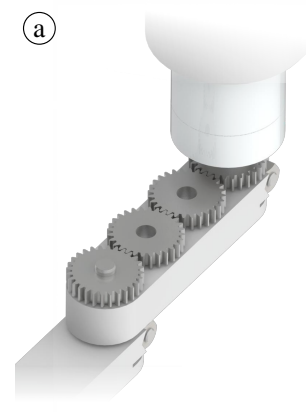

(b)
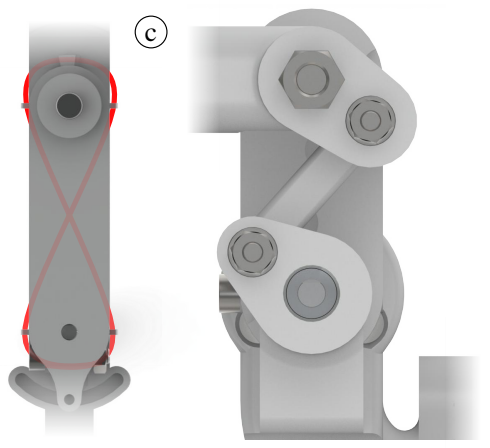

Fig. 4. Different linkage designs for the mapping of the dependent joints; (a) gear-based design, (b) tendon-based design, and (c) currently implemented four-bar linkage design.

at the end effector that the user holds and manipulates the device. The gripper consists of two spring-loaded passive joints coupled together with a gear mechanism. A small neodymium magnet is embedded in one lever. The grip angle can be measured using a Hall effect sensor integrated in the middle of the end effector. A Mini40 6-DoF force/torque sensor (ATI Industrial Automation, USA) is integrated at the end effector to assist in the friction compensation of certain joints, as presented in section III-C. The detailed assembly of the finger grips is presented in Fig. 3.

Different approaches to emulate the behaviour of the instrument's dependent joints were explored, as presented in Fig. 4. The first method uses a series of gears placed along the link to couple the joint angle from the active joint to the passive one. This can be implemented using mostly off-the-shelf components. However, it limits the possible combinations of link lengths due to the limited availability of gear pitch and number of teeth. Additionally, this can only be done with an even numbers of gears to achieve the correct rotational direction. Adding multiple gears can also introduce significant backlash to the system. Alternatively, a crosstendon mechanism can be used instead of gearing. This can be implemented in arbitrary link lengths. Nevertheless, it still suffers from the hysteresis characteristics of the tendon, and the increased complexity of the assembly process. Finally, a four-bar mechanism was chosen for the proposed master manipulator because it does not suffer from the drawbacks of the former approaches. It further provides more rigidity with comparable size. Fig. 5 depicts the schematic of the mechanism. The primary link length $l$ is fixed by the desired length of the manipulator's scaling factor, as previously described. In addition, two symmetric fixtures with length $a$ are added to each side of the link with an angle $\theta$. The second bar connects the fixtures diagonally via bearings. According to the geometry, this linkage produces a small angular deviation $\epsilon$ from an ideal dependent joint, where both joint angles are equal. This error is minimised by choosing $\theta$ to be as small as possible while taking into account the space required by the hinge joints. As presented in Fig. 5, for a given fixture length $a$ an angle $\theta$ can be calculated which
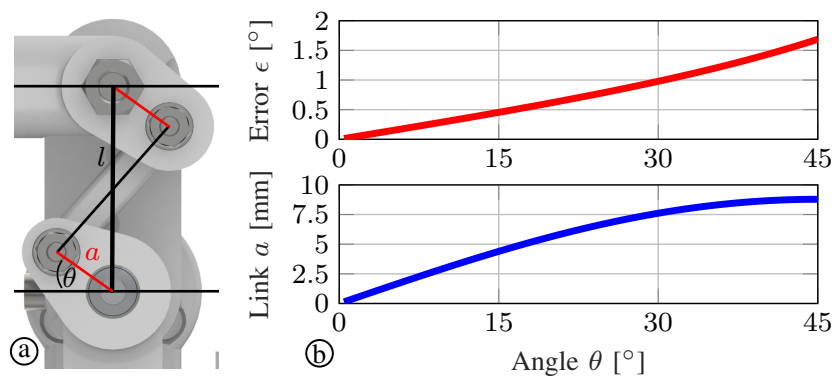

Fig. 5. Parameter optimisation of the four bar mechanism. a.) parameter definition of the mechanism. b.) blue: relation between angle $\theta$ and linklength $a$, red: angular error $\epsilon$ as function of $\theta$.

results into a maximal orientation error $\epsilon$ :

$$
\epsilon(\theta)=\max _{q}\left\{\operatorname{abs}(\tilde{q}(\theta, q)-q) \mid q \in\left[q^{\min }, q^{\max }\right]\right\}
$$

where $q$ denotes the desired joint angle, $\tilde{q}$ is the resulting joint angle, and $q^{\min }, q^{\max }$ are the ranges of joint motion.

Hence, the constrained optimisation problem can be formulated as:

$$
\begin{array}{rlrl}
\theta^{\star} & =\arg \min (\epsilon(\theta)) \\
\text { subject to } & a \geq a_{\min } \\
\text { and } \quad & \theta & =f^{-1}(a)
\end{array}
$$

where $a_{\min }$ represents the mechanical constraints and $f^{-1}$ is the function that relates the fixtures length to the angle $\theta$. The optimal solution $\theta^{\star}$ was calculated as $\theta^{\star}=35^{\circ}$ resulting in maximal angular errors of $\epsilon=1.2^{\circ}$, for $l=30 \mathrm{~mm}$.

\section{Workspace Analysis}

The controlled surgical instruments have workspace limitations due to their geometry and their joint limits. It is important for the user to be able to percieve these limitations so that they can adapt their manipulation strategy accordingly, and retain an intuitive control of the instruments even at the edge of the workspace. These limitations can be conveyed implicitly to the user by providing them with an input device possessing similar properties. To compare the workspace of the slave and the master device, a workspace analysis was performed examining the dexterous workspace of their respective manipulators. A master device with a highly dexterous workspace may give the user the impression that certain instrument poses are feasible although they are not. Conversely, a master workspace with a small dexterous workspace may needlessly constrain the user's motions.

The dexterity measure $\mathcal{D}$ is calculated following the standard techniques of calculating the manipulability measure $\mathcal{M}$, introduced in [9]:

$$
\mathcal{M}=\sqrt{\left|\mathbf{J} \mathbf{J}^{T}\right|}
$$

where $\mathbf{J}$ is the end effector Jacobian matrix. Since $\mathcal{M}$ does not consider mechanical constraints of the manipulator the dexterity measure is calculated as:

$$
\mathcal{D}=\sqrt{\left|\mathbf{J}^{q} \mathbf{J}^{q^{T}}\right|},
$$



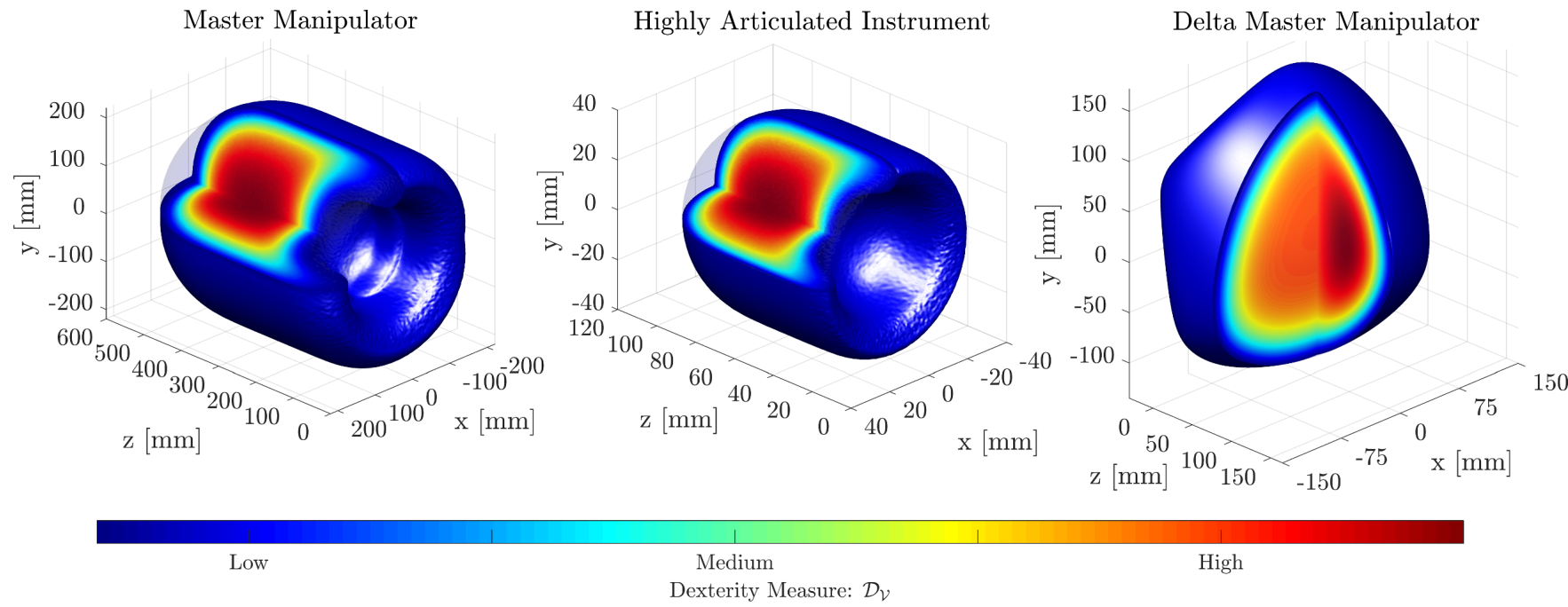

Fig. 6. Dexterous workspace $\mathcal{D} \mathcal{V}$ of the master manipulators and the highly articulated instrument.

where $\mathbf{J}^{q}$ is the joint-limit constrained end effector Jacobian. Using a constrained Jacobian to calculate the dexterity of a manipulator formalises the effects of the joint limits. Unlike in [10], the individual columns of $\mathbf{J}$ are penalised. When the $i^{\text {th }}$ joint-value $q_{i}$ approaches the limits $q_{i, \min }$ or $q_{i, \max }$ as:

$$
\mathcal{P}_{i}^{q}=\frac{1-\exp \left\{\frac{4 \kappa_{q}\left(q_{i}-q_{i, \min }\right)\left(q_{i, \max }-q_{i}\right)}{\left(q_{i, \max }-q_{i, \min }\right)^{2}}\right\}}{1-\exp \left\{\kappa_{q}\right\}},
$$

where the factor " 4 " and the denominator term " $1-\exp \left\{\kappa_{q}\right\}$ " in (7) are needed to normalise the penalisation term such that $\mathcal{P}_{i}^{q}$ spans the interval $[0,1]$. At the joint-limits $\mathcal{P}_{i}^{q}$ evaluates to zero, and in the neutral position $\mathcal{P}_{i}^{q}$ evaluates to one. The scaling coefficient $\kappa_{q}$ specifies the functional shape in between these points. The constrained Jacobian $\mathbf{J}^{q}$ is formed by penalising the columns $\mathbf{j}_{i}^{q}$ individually by

$$
\mathbf{j}_{i}^{q}=\mathcal{P}_{i}^{q} \mathbf{j}_{i}^{e},
$$

where $\mathbf{j}_{i}^{e}$ is the $i^{\text {th }}$ column of the end effector Jacobian. In contrast to the global penalisation presented in [10], this presented joint-wise penalisation is also suitable for redundant robots. The dexterity measure presented in [10] evaluates to zero when one joint reaches the limit, which is not appropriate for a redundant robot whose kinematics would not degenerate to an underconstrained system.

Multiple configurations (q) can map to the same end effector position $(\mathbf{x})$. The discretised workspace $\mathcal{V}$ which is comprised of voxels $v(i, j, k) \in \mathbb{R}^{3}$ and where $i, j, k$ denote the indices of the voxels, is calculated as:

$$
\mathcal{D}_{\mathcal{V}}(i, j, k)=\max _{\mathbf{q}_{m}}\left\{\mathcal{D}\left(\mathbf{q}_{m}\right) \mid \mathbf{x}\left(\mathbf{q}_{m}\right) \in v(i, j, k)\right\} .
$$

A comparison of dexterous workspaces is depicted in Fig. 6. The computed dexterous workspaces illustrate the described dexterity measure $\mathcal{D}_{\mathcal{V}}$ of i.) the proposed master manipulator, ii.) the slave instruments manipulated by the master device, and iii.) the previously used master manipulator, the omega.7 delta robot. The visualisation illustrates that the omega.7 workspace shape is not suitable to manipulate the highly articulated tools designed for working in confined cylindrical shaped workspaces. In contrast, the dexterous workspace of the optimised master manipulator closely maps the workspace of the instruments.

\section{Control SyStem}

\section{A. Joint Limit Force Rendering}

To inform the user about when joint-limits are approached, a smooth haptic rendering profile is employed, rendering an increasing resistance the closer the user comes to the limits. Assuming symmetrical joint-limits of $\pm q_{i}^{\lim }$, the motor torque for the $i^{t h}$ joint is calculated as:

$$
\begin{aligned}
q_{i}^{\lim , l} & :=q_{i}^{\lim }-q_{i}^{\text {stiff }} \\
\tau_{i}\left(q_{i}\right) & =\tau_{i, \max } \cdot \\
& \begin{cases}0 & , \text { if }\left|q_{i}\right|<q_{i}^{\lim , l} \\
1 & , \text { if }\left|q_{i}\right|>q_{i}^{\lim } \\
\frac{1}{2}-\frac{1}{2} \cos \left(\pi \frac{\left(\left|q_{i}\right|-q_{i}^{\lim , l}\right)}{q_{i}^{\text {stiff }}}\right) & , \text { else }\end{cases}
\end{aligned}
$$

where $q_{i}^{\lim , l}$ is the lower limit from which the torque rendering ramps up. The stiffness is denoted as $q_{i}^{\text {stiff }}$, and $\tau_{i, \max }$ is the maximum torque rendered.

\section{B. Gravity Compensation}

The manipulator has a serial-link structure with integrated actuators and sensors in its joints. This results in a highly unbalanced and non-uniform variation in the weight perceived at the finger grip when it is manipulated throughout its workspace, which makes it unnatural to be manoeuvred. Additionally, the weight always pulls the device downward when the user releases the manipulator. The torque at each joint exerted due to gravity can be determined using the known mass parameters from the CAD design. The undesired weight can be compensated by providing the same amount of counter-torque at each joint. The parameters used for 
modelling the gravity torque include the mass and centre of mass of the links. The gravity torque is obtained by computing the inverse dynamics of the manipulator at zero velocity with acceleration equals to the gravity. A performance-optimised implementation of recursive NewtonEuler method by [11] is adopted to compute the inverse dynamics. Although the accuracy of the mass parameters calculated from the mechanical design are acceptable, the effect of the residual gravity force is further reduced by additional viscous damping:

$$
\tau_{d}=-k \dot{q}
$$

where $k$ defines the damping coefficient, $\dot{q}$ denotes the joint velocity, and $\tau_{d}$ is the damping torque provided by the actuator. The damping can also reduce the abrupt motion by presenting additional friction to the user when the excessive manipulation speed is applied.

\section{Friction Compensation}

A significant obstacle to the use of serial-link master manipulators for joint space control is the restrictions this approach imposes on the structure of the master device. This is particularly noticeable for devices with a high number of DoFs. In this paper, the kinematic structure of the instruments imposes that the first two joints of the master manipulator be the translation and roll joints. However, the user only grasps the master manipulator at the end effector, on the very last joint.

The roll joint possesses a higher gear reduction (262:1, as opposed to $16: 1$ or $35: 1$ for the other joints), and far less cantilever advantage than any other rotational joints. In practice, and as shown in section IV-A, the materials used and friction present in the device mean that it is impossible for the user to rotate the roll joint without having to exert prohibitively high torques on the end effector. The translational joint encounters a similar problem as the translation forces are generated offaxis, thus creating torques hampering those same forces from translating the linear stage.

To address these issues, a 6-DoF force/torque sensor was incorporated in the last joint. The forces and torques detected by the sensor are transformed into the base joint's frame of reference following:

$$
\begin{aligned}
{ }^{B} F_{S} & =T_{E}{ }^{E} T_{S} F_{S}, \\
{ }^{B} T q_{S} & =T_{E}{ }^{E} T_{S} T q_{S},
\end{aligned}
$$

where $F_{S}$ and $T q_{S}$ are the forces and torques read from the sensor, ${ }^{B} F_{S}$ and ${ }^{B} T q_{S}$ are the forces and torques expressed in the base joint frame of reference, $T_{E}$ is the homogeneous transform from the base joint to the end effector as obtained from the forward kinematics, and ${ }^{E} T_{S}$ the static transformation from the end effector to the sensor frame of reference.

Torques detected by the sensor along the roll axis are then used to generate additional torques in the roll joint, using a proportional law with a dead band. Likewise, forces detected by the sensor along the translation axis are used to generate additional forces in the linear stage.

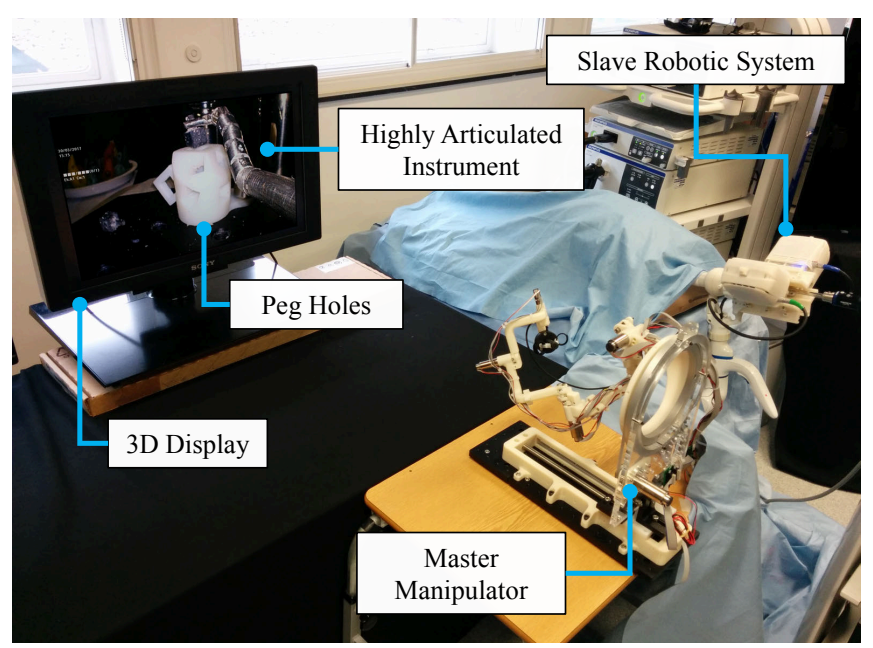

Fig. 7. Experimental setup of the usability study with the peg insertion task, presenting the master manipulator and the slave robotic system for single access robotic surgery.

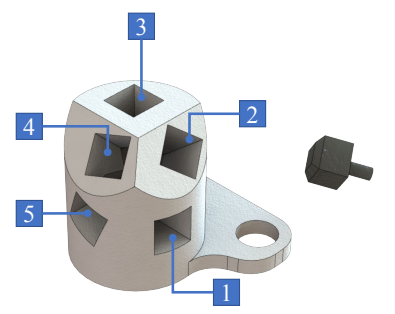

Fig. 8. Structure and a peg for the insertion task, showing five different locations of the holes and the rubber peg.

\section{EXPERIMENTAL RESULTS}

\section{A. Gravity and Friction Compensations}

Evaluation of the gravity and friction compensation methods was carried out using the force/torque sensor integrated at the end effector. This sensor can directly measure the forces applied perceived by the user at the finger grips. Two short sequences of movements were performed by a user, each with one of two different control schemes applied to the manipulator. In one case no kind of compensation was used, and in the other both gravity and friction compensation were used. An excerpt of the experimental results are presented in Fig. 9. In the first case, where the gravity compensation and friction compensation methods are used, the maximum force and torque are $2.1 \mathrm{~N}$ and $42 \mathrm{Nmm}$ with mean values of $0.9 \mathrm{~N}$ and $19 \mathrm{Nmm}$ respectively. In contrast, without the compensations, the maximum force and torque exerted are 6.4 $\mathrm{N}$ and $151 \mathrm{Nmm}$ with mean values of $3.1 \mathrm{~N}$ and $78 \mathrm{Nmm}$. This shows a significant amount of reduction in terms of the effort required by the user to move the manipulator. Furthermore, without the friction compensation, the movements of the translation stage and the roll joint are very limited.

\section{B. Usability Study}

An experimental setup, presented in Fig. 7, was used to study the usability of the proposed manipulator in comparison with delta manipulators: omega.7 and sigma.7. Five 

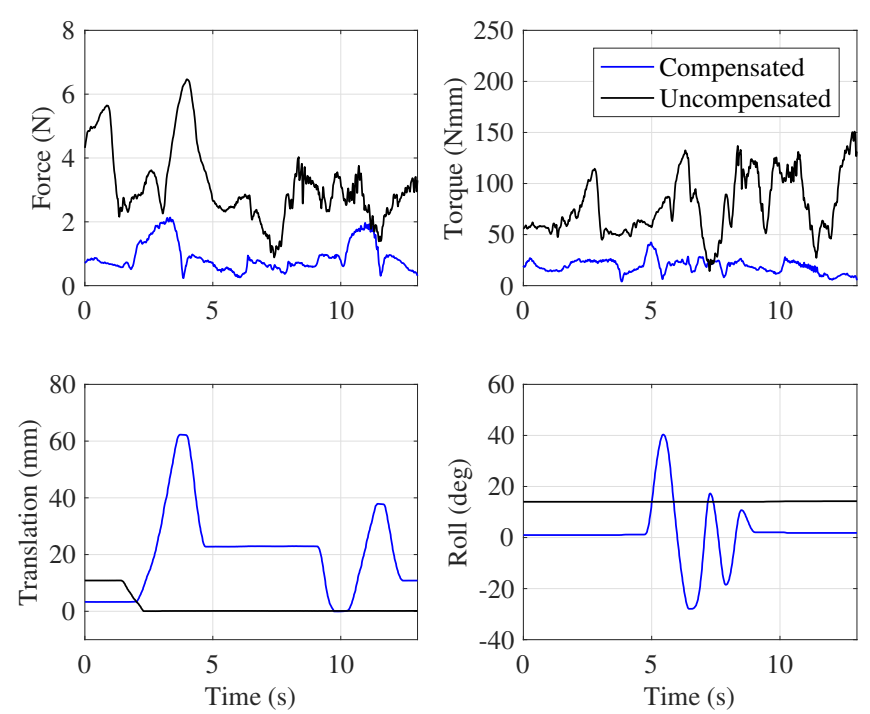

Fig. 9. Comparison of manipulation force/torque and the movement of the translation/roll joints in implementations with and without the friction and gravity compensations.

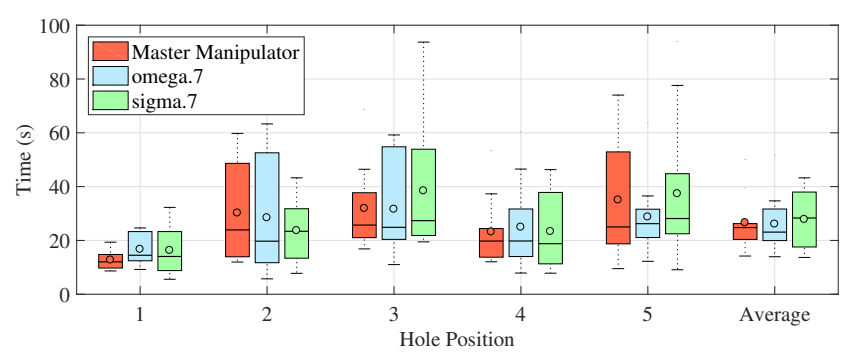

Fig. 10. Task execution times between the master manipulators. The mean values are indicated by the circles.

participants performed a single-handed peg insertion task into predefined holes in a fixed order using a structure and a peg shown in Fig. 8. All the participants had prior experience in using the robotic surgical system with delta manipulators. The experiment was repeated twice for each user, and the time between each successful insertion was recorded. Fig. 10 presents the summary of the performance of the task. The number of times the delta manipulators had to be clutched are 28 and 12 for the omega. 7 and sigma.7 respectively.

\section{CONCLUSION}

This paper presents a joint space master manipulator device, designed for the control of highly articulated surgical instruments. The motivation for the development of this manipulator stemmed from the authors' past experience using task space master manipulators with highly articulated surgical instruments. In particular, the need for clutching and lack of intuitive access to joint information proved to be two major drawbacks of these manipulators. The presented system solves both these issues. The advantage of not having to rely on optimisation-based inverse kinematic approaches further increases the ease with which the system can be handled. The system can be safely driven to multiple joint limits, and still remain intuitively controlled as the limits are clearly marked using force feedback. The comparative usability study with other delta manipulators shows that the proposed master manipulator can perform equally well in the peg manipulation task, without clutching.

The main challenge involved with this approach is to retain the ergonomics of an optimised task-space manipulator, such as an omega.7, while using a mechanical structure not optimised to that effect. The use of gravity compensation, and particularly force-torque compensation, has been instrumental in achieving this goal. However, several aspects present scope for further improvement. As the current device is at the prototype stage, a large number of components are 3D printed and lack stiffness. Combined with the fact that the model used for the gravity compensation was determined from theoretical values, the resulting gravity compensation, while sufficient, can still be improved. This could be achieved using a mass parameters calibration method such as [12]. A side effect of this imperfect compensation is to require the user to exert small forces to maintain the master manipulator in a given position. As a result, the force sensor may detect forces that do not really represent the user's intention to move, and slightly distort the force-based motions in certain joint configurations. While non-critical, addressing these calibration and compensation issues will improve the usability of the system, further highlighting the advantages derived from this approach.

\section{REFERENCES}

[1] S. Matich et al., "A new single-port robotic system based on a parallel kinematic structure," in 2015 IEEE/RSJ International Conference on Intelligent Robots and Systems, Hamburg, 2015, pp. 236-241.

[2] J. Shang et al., "A Single-Port Robotic System for Transanal Microsurgery-Design and Validation," IEEE Robotics and Automation Letters, vol. 2, no. 3, pp. 1510-1517, 2017.

[3] K. Leibrandt et al., "Effective Manipulation in Confined Spaces of Highly Articulated Robotic Instruments for Single Access Surgery," IEEE Robotics and Automation Letters, vol. 2, no. 3, pp. 1704-1711, 2017.

[4] S. J. Phee et al., "Master and slave transluminal endoscopic robot (MASTER) for natural orifice transluminal endoscopic surgery (NOTES)," in Annual International Conference of the IEEE Engineering in Medicine and Biology Society, Minneapolis, MN, 2009, pp. 1192-1195.

[5] S. Shim et al., "An all-joint-control master device for single-port laparoscopic surgery robots," International Journal of Computer Assisted Radiology and Surgery, vol. 11, no. 8, pp. 1547-1557, 2016.

[6] C. Neupert et al., "Pseudo-Haptic Feedback in Teleoperation," IEEE Transactions on Haptics, vol. 9, no. 3, pp. 397-408, 2016.

[7] Z. M. Thant et al., "Ergonomic master controller for flexible endoscopic gastrointestinal robot manipulator," in International Conference on Biomedical and Pharmaceutical Engineering, Singapore, 2006, pp. $575-579$.

[8] C. A. Seneci et al., "Design of a Smart 3D-printed Wristed Robotic Surgical Instrument with Embedded Force Sensing and Modularity," in IEEE International Conference on Intelligent Robots and Systems, Daejeon, 2016, pp. 3677-3683.

[9] T. Yoshikawa, "Manipulability of Robotic Mechanisms," The International Journal of Robotics Research, vol. 4, no. 2, pp. 3-9, 1985.

[10] M. J. Tsai and Y. H. Chiou, "Manipulability of manipulators," Mechanism and Machine Theory, vol. 25, no. 5, pp. 575-585, 1990.

[11] P. I. Corke, Robotics, Vision \& Control: Fundamental Algorithms in Matlab. Springer, 2011.

[12] D. Ma and J. Hollerbach, "Identifying mass parameters for gravity compensation and automatic torque sensor calibration," in IEEE International Conference on Robotics and Automation, Minneapolis, MN, 1996, pp. 661-666. 